\title{
ALGEBRAIC HULLS AND SMOOTH ORBIT EQUIVALENCE
}

\author{
ALESSANDRA IOZZI
}

\begin{abstract}
For $i=1,2$, let $\mathscr{F}_{i}$ be foliations on smooth manifolds $M_{i}$ determined by the actions of connected Lie groups $H_{i}$; we describe here some results which provide an obstruction, in terms of a geometric invariant of the actions, to the existence of a diffeomorphism between the $\mathscr{F}_{i}$ 's.
\end{abstract}

\section{INTRODUCTION}

Let $\left(M_{i}, \mu_{i}\right), i=1,2$, be two manifolds with quasi-invariant measures, and let $H_{i} \subset \operatorname{Diff}\left(M_{i}\right)$ be connected Lie groups. If there is a measure class preserving diffeomorphism $\theta: M_{1} \rightarrow M_{2}$ which is a bijection of $H_{1}$-orbits and $\mathrm{H}_{2}$-orbits then we say that the actions are smoothly orbit equivalent. If the $H_{i}$-actions determine foliations $\mathscr{F}_{i}$ on the manifolds $M_{i}$, then the map $\theta$ is just a diffeomorphism between the foliations. These phenomena, and the corresponding phenomena arising in situations in which the $\left(M_{i}, \mu_{i}\right)$ are just Borel (or topological) $H_{i}$-spaces and the map $\theta$ is a measure class preserving Borel isomorphism (or homeomorphism), have been studied, using a variety of techniques, independently by several authors [B, CFW, D1, D2, K, PnZ, W1, W2, Z2, Z4]. Most of the results obtained require some strengthening of the hypotheses, such as finiteness and invariance of the measures and amenability or semisimplicity (in higher rank) of the groups acting.

One of the results that we want to describe in this paper fits in this geometric setting and provides an obstruction, in terms of a geometric invariant of the actions, to the foliations being diffeomorphic. Recall that, if $H$ acts ergodically and by diffeomorphisms on the $n$-dimensional manifold $M$, the algebraic hull of the $H$-action is the unique (up to conjugacy) smallest algebraic subgroup $L \subset \mathrm{GL}(n, \mathbf{R})$ such that there exists a measurable $H$-invariant reduction to $L$ of the frame bundle on $M$, on which $H$ acts by automorphisms. (For an analytic definition see $\S 2$ and for the general context see [Z5, 9.2].) Then the normal algebraic hull of the $H$-action on $M$ will be the projection of $L$ in the direction normal to the orbits.

Theorem 4.1. Let $H_{1}, H_{2}$ be connected Lie groups, $H_{i} \subset \operatorname{Diff}\left(M_{i}\right)$, where $M_{i}$ are smooth manifolds with a quasi-invariant measure $\mu_{i}$. Suppose that the

Received by the editors June 17, 1989 .

1980 Mathematics Subject Classification (1985 Revision). Primary 22D40, 22E40, 57Sxx, 28D99, $54 \mathrm{H} 20,58 \mathrm{~F} 18$. 
actions are locally free, essentially free (i.e., free on a conull set) and ergodic, and let $\mathscr{F}_{i}$ be the corresponding foliations. If the $\mathscr{F}_{i}$ are diffeomorphic, then the normal algebraic hulls are conjugate.

Notice that there are no assumptions on the finiteness or the invariance of the measures; moreover local freeness is necessary only to be able to state the theorem in terms of foliations (instead of orbits), and we require ergodicity only to state the result in terms of algebraic hulls globally and not restricting the attention to each ergodic component.

Many interesting examples arise as particular cases of homogeneous spaces $G / \Gamma$, where $G$ is a connected, semisimple Lie group with no compact factors and $\Gamma$ is a lattice in $G$; the action of $H$ will hence be via an embedding $H \hookrightarrow G$, and the foliation determined by the $H$-action will be identified with the double cosets $H \backslash G / \Gamma$. (Notice that, in this context, essential freeness of the $H$-action on $M=G / \Gamma$ follows automatically, at least in the case in which $H$ is algebraic, as it is shown in Proposition 4.4.) This situation has been extensively studied, not only in the case in which $G$ is semisimple, [B, M, Pr, Rt, $\mathrm{W} 1, \mathrm{~W} 2]$. One of the most recent and complete results is due to Dave Witte [W2]; generalizing an idea of D. Benardete [B], he proved that any (continuous) orbit equivalence between two foliations $H_{i} \backslash G_{i} / \Gamma_{i}$ (where the $G_{i}$ 's are semisimple groups to which the Mostow Rigidity theorem applies or suitable solvable groups or simply connected nilpotent groups) is the composition of an affine map and a homeomorphism that preserves each leaf, provided that the $H_{i}$ 's are unimodular. (An affine map between $G_{1} / \Gamma_{1}$ and $G_{2} / \Gamma_{2}$ is the composition of a homomorphism $\sigma: G_{1} \rightarrow G_{2}$, such that $\sigma\left(\Gamma_{1}\right) \subset \Gamma_{2}$, and a translation.) Our approach to the problem is completely different and, for this kind of action, the next theorem provides an explicit way to compute the algebraic hulls.

Theorem 3.1. Let $G$ be a connected semisimple Lie group with no compact factors and finite center, $\Gamma \subset G$ an irreducible lattice and $H \subset G$ a noncompact closed subgroup. Then the algebraic hull of the $H$-action on $G / \Gamma$ is the algebraic hull of $\operatorname{Ad}_{G}(H)$.

It is worth observing here that, building on Theorems 4.1 and 3.1, our methods give results for the group $H=a x+b \simeq \mathbf{R}^{\times} \ltimes \mathbf{R}$ of affine motions of the line which do not seem to be accessible so far by other techniques (see $\S 4$ for details). Moreover, besides being of independent interest, this theorem also gives results in terms of smoothly conjugate actions. In fact we have

Corollary 3.2'. Let $G, \Gamma$ be as above, and let $H$ be a noncompact Lie group acting on $G / \Gamma$ via two different embeddings $\pi_{1}, \pi_{2}: H \rightarrow G$ with closed images $H_{1}, H_{2}$. If the actions of $H$ on $G / \Gamma$ given by $\pi_{i}$ 's are conjugate, then $\operatorname{ad}_{\mathfrak{g}}\left(\mathfrak{h}_{1}\right)$ and $\operatorname{ad}_{\mathfrak{g}}\left(\mathfrak{h}_{2}\right)$ are conjugate as subalgebras of $\mathfrak{g l}(\mathfrak{g})$.

It needs to be recalled here that a much stronger result (even though with a narrower range of application in the semisimple case) has been proven by 
D. Witte [W1]; he showed, in fact, that any measurable isomorphism of ergodic unipotent translations on $G / \Gamma$ (where $G$ is a connected Lie group and $\Gamma$ is any closed subgroup of $G$ such that $G / \Gamma$ has finite volume) is an affine map, or, in other words, that $H_{1}$ and $H_{2}$ are conjugate in $G$. (A weaker conclusion holds in the case in which the $H_{i}$ 's have zero-entropy.) In $\S 3$ we shall illustrate how to deal with some examples which can be approached both using our result and Witte's theorem.

The proof of these results are essentially based on two ingredients: the first is a correspondence in cohomology between the cocycle of the $\mathrm{H}$-action and a cocycle of a suitable $\Gamma$-action, ([Z3] and Lemma 3.1); the second ingredient is the correspondence (under the necessary hypotheses) between the algebraic hull of a $G$-action and the algebraic hull of the restriction of the action to $\Gamma$ (Theorem 2.1). It should be noticed here that the necessity of restricting our attention to semisimple groups is due to the fact that Theorem 2.1 is known, so far, only in this case.

For completeness, although in a different spirit, we study also the case in which $G$ is a compact Lie group, and the following theorem is proven in $\S 3$.

Theorem 3.4. Let $H, G$ be Lie groups with $G$ compact, and let $\pi_{i}: H \rightarrow G$, $i=1,2$, be two embeddings with dense image $\pi_{i}(H)=H_{i}$. If the actions of $H_{i}$ 's on $G$ are measurably conjugate, then there exists an automorphism $A$ of $G$ such that $A\left(H_{1}\right)=H_{2}$.

I want to thank my advisor, R. Zimmer, for many useful conversations we had during the preparation of this paper, which was part of my Ph.D. thesis at the University of Chicago.

\section{Preliminaries on Cocycles}

Let $(S, \mu)$ be a $G$-space with a quasi-invariant measure $\mu$, and let $\alpha, \beta: S \times$ $G \rightarrow H$ be measurable cocycles. Recall that we say that $\alpha$ is equivalent to $\beta$ (and we write $\alpha \sim \beta$ ) if there exists a measurable map $\varphi: S \rightarrow H$ such that, for every $g \in G, \beta(s, g)=\varphi(g s) \alpha(s, g) \varphi(s)^{-1}$ for almost every $s \in S$. If $G$ is ergodic on $S$ and $H$ is algebraic there exists a unique (up to conjugacy) smallest algebraic subgroup $L \subseteq H$ such that $\alpha$ is equivalent to a cocycle taking values in $L$ but is not equivalent to a cocycle taking value in a proper algebraic subgroup of $L$. It follows that if $\alpha \sim \beta, \beta(S \times G) \subseteq J$ and $J$ is algebraic, then $J$ contains a conjugate of $L$. The conjugacy class of $L$ is called the algebraic hull of $\alpha$ [Z5, 9.2]. Given any group $G$ acting by diffeomorphisms on a smooth manifold $M$, we define the algebraic hull of the $G$-action on $M$ to be the algebraic hull of the derivative cocycle $\alpha: M \times G \rightarrow \mathrm{GL}(n, \mathbf{R})$ defined as $\alpha(m, g)=(d g)_{m}$, after a measurable trivialization of the tangent bundle.

Let $\alpha_{\Gamma}: S \times \Gamma \rightarrow H$ denote the restriction of a cocycle $\alpha$ to $\Gamma$. The next result will be crucial in proving Theorems 3.1 and 4.1 .

Theorem 2.1 [I]. Let $G$ be a connected semisimple Lie group with finite center and no compact factors, and let $\Gamma \subset G$ be an irreducible lattice. Let $\alpha: S \times G \rightarrow$ $H$ be a cocycle of an ergodic $G$-action into an algebraic group, and assume that 
the G-action of $G$ on $S$ is not essentially transitive with compact stabilizers. Then the algebraic hulls of $\alpha$ and $\alpha_{\Gamma}$ are the same.

Recall that, under the above hypotheses on $G, \Gamma$ and $S$, the restriction to $\Gamma$ of the $G$-action is still ergodic [Mo, $\mathrm{Z} 1$ ]; this implies in turn that the product action of $G$ on $S \times G / \Gamma$ is ergodic as well [Z3, Proposition 2.2.2].

Let $Z(S \times G ; H)$ be the set of cocycles from $S \times G$ into $H$, and let $H^{1}(S \times G ; H)$ be the set of the corresponding equivalence classes of cocycles.

Proposition 2.2. Let $G, \Gamma, H$ and $S$ be as above. Then there is a bijection in cohomology $\Psi: H^{1}(S \times \Gamma ; H) \rightarrow H^{1}(S \times G / \Gamma \times G ; H)$ which preserves the algebraic hulls.

Remark. The existence of the bijection holds in much greater generality [Z3] for any closed subgroup $\Gamma$ of a locally compact topological group $G$, any locally compact topological group $H$ and any $G$-space $S$. However, besides the fact that the above situation is the one in which we shall use this result, the correspondence between algebraic hulls makes sense only under suitable hypotheses which insure ergodicity of all the actions.

Proof. Since we shall use it in the sequel, we give here the explicit form of this correspondence, leaving out the details. For a complete proof see [I, Z3]. Let $\eta: G / \Gamma \rightarrow G$ be a Borel section of the canonical projection $p: G \rightarrow G / \Gamma$ such that $\eta(p(g)) g \in \Gamma$, for every $g \in G$. Then, if $[\alpha] \in H^{1}(S \times \Gamma ; H)$ and $\alpha \in$ $Z(S \times \Gamma ; H)$ with $\alpha \in[\alpha]$, we define $\Psi(\alpha)(s, x, g)=\alpha\left(\eta(x), \eta(g x) g \eta(x)^{-1}\right)$; the equivalence class of $\Psi(\alpha)$ will be independent of the choice of $\alpha \in[\alpha]$, and hence $\Psi$ will be a map in cohomology. Conversely, given

$$
[\beta] \in H^{1}(S \times G / \Gamma \times G ; H)
$$

and $\beta \in[\beta]$, we want to define $\Phi=\Psi^{-1}$; choose $x \in g[e] \in G / \Gamma$ and define $\Phi(\beta) \in Z(S \times \Gamma ; H)$ as $\Phi(\beta)(s, \gamma)=\beta\left(g s, x, g \gamma g^{-1}\right)$. Then $\Psi(\beta)$ is independent in cohomology of the choice of $x \in G / \Gamma, \Phi$ and $\Psi$ are inverses in cohomology and both maps preserve the algebraic hulls.

Let $\left(S_{i}, \mu_{i}\right)$ be $H_{i}$-spaces, $i=1,2$; we say that the actions of $H_{1}$ and $\mathrm{H}_{2}$ are orbit equivalent if there exists a measure class preserving Borel isomorphism (possibly after discarding a set of measure zero) $\theta: S_{1} \rightarrow S_{2}$ that takes any $H_{1}$-orbit onto an $H_{2}$-orbit. If the $H_{2}$-action is essentially free then the map $\theta$ defines a cocycle $\lambda: S_{1} \times H_{1} \rightarrow H_{2}$ which satisfies the relation $\theta\left(h_{1} s_{1}\right)=\lambda\left(s_{1}, h_{1}\right) \theta\left(h_{1}\right)$. If the cocycle $\lambda$ is equivalent to a cocycle independent of $s_{1} \in S_{1}$, namely, if $\lambda \sim \lambda_{\rho}$ where $\lambda_{\rho}\left(s_{1}, h_{1}\right)=\rho\left(h_{1}\right)$ with $\rho: H_{1} \rightarrow H_{2}$ a homomorphism, then we say that the actions are conjugate (or isomorphic). If $S_{1}$ and $S_{2}$ are manifolds and the map $\theta$ is a diffeomorphism then we say that the actions are respectively smoothly orbit equivalent and smoothly conjugate (or smoothly isomorphic); moreover, in the essentially free case, the cocycle $\lambda$ 
will be smooth along a.e. orbit. The correspondence in cohomology described in the next proposition is well known $[\mathrm{Rm}]$ and we shall use this result several times.

Proposition 2.3. IF $\theta: S_{1} \rightarrow S_{2}$ is an orbit equivalence between essentially free spaces, then there is a bijection $\Upsilon: Z\left(S_{2} \times H_{2} ; L\right) \rightarrow Z\left(S_{1} \times H_{1} ; L\right)$ which is a bijection in cohomology and preserves the algebraic hulls.

Proof. If $\beta \in Z\left(S_{2} \times H_{2} ; L\right)$, let us define $\tilde{\beta} \in Z\left(S_{1} \times H_{1} ; L\right)$ as $\tilde{\beta}\left(s_{1}, h_{1}\right)=$ $\Upsilon(\beta)\left(s_{1}, h_{1}\right)=\beta\left(\theta\left(s_{1}\right), \lambda\left(s_{1}, h_{1}\right)\right)$, where $\lambda: S_{1} \times H_{1} \rightarrow H_{2}$ is the cocycle associated to $\theta$. Conversely, if $\alpha \in Z\left(S_{1} \times H_{1} ; L\right)$, let us define $\Xi(\alpha)=\alpha_{0} \in$ $Z\left(S_{2} \times H_{2} ; L\right)$ as $\alpha_{0}\left(s_{2}, h_{2}\right)=\alpha\left(\theta^{-1}\left(s_{2}\right), \eta\left(s_{2}, h_{2}\right)\right)$, where $\eta: S_{2} \times H_{2} \rightarrow H_{1}$ is the cocycle associated to $\theta^{-1}$; namely, if $\lambda\left(s_{1}, h_{1}\right)=h_{2}$ and $\theta\left(s_{1}\right)=s_{2}$, then $\eta\left(s_{2}, h_{2}\right)=\eta\left(\theta\left(s_{1}\right), \lambda\left(s_{1}, h_{1}\right)\right)=h_{1}$. It is straightforward to verify that the maps $\Xi$ and $\Upsilon$ are inverses and that equivalence classes of cocycles, as well as algebraic hulls, are preserved.

\section{SMOOThly CONJUGATE ACtIONS}

Let $G$ be a connected semisimple Lie group with no compact factors, finite center and Lie algebra $\mathfrak{g}$, and let $\Gamma$ be an irreducible lattice in $G$. Let $H$ be a noncompact Lie group and let $\pi_{1}, \pi_{2}: H \rightarrow G$ be two different embeddings of $H$ with closed images $H_{i}=\pi_{i}(H), i=1,2$.

The first result we want to prove will be a very easy consequence of the next theorem which illustrates how to compute the algebraic hull of the $\mathrm{H}$-action on $G / \Gamma$ by diffeomorphisms, where, for simplicity of notation, we identify $H$ with its embedded image in $G$.

Recall that, since $\Gamma$ is discrete, the tangent bundle $\mathrm{T}(G / \Gamma)$ can be smoothly identified with $G / \Gamma \times \mathfrak{g}$. Then the action of $H \subset G \subset \operatorname{Diff}(G / \Gamma)$ on $\mathrm{T}(G / \Gamma)$ is given, via this identification, by the derivative cocycle $\alpha: G / \Gamma \times H \rightarrow \mathrm{GL}(\mathfrak{g})$, $\alpha(x, h)=(d h)_{x}=\operatorname{Ad}_{G}(h)$, for $x \in G / \Gamma$ and $h \in H$.

Theorem 3.1. Let $G$ be a semisimple noncompact Lie group with no compact factors and finite center, $\Gamma \subset G$ an irreducible lattice and $H \subset G$ a noncompact closed subgroup. Then the algebraic hull of the $H$-action on $G / \Gamma$ is the algebraic hull of $\operatorname{Ad}_{G}(H)$.

Before proving this theorem note the following consequence.

Corollary 3.2. Let $G, \Gamma, H_{i}, i=1,2$, be as above. If the $H_{1}$-action and the $H_{2}$-action on $G / \Gamma$ are smoothly isomorphic, then the algebraic hulls of $\operatorname{Ad}_{G}\left(H_{1}\right)$ and $\operatorname{Ad}_{G}\left(H_{2}\right)$ are conjugate as subgroups of $\mathrm{GL}(\mathfrak{g})$.

Remarks. (1) If $H$ is a noncompact connected simple Lie group, the result of Theorem 3.1 is in [Z6, Corollary 5.3] and is a consequence of the Borel density theorem.

(2) A natural question is to ask whether or not Theorem 3.1 is true for subgroups $\Gamma$ which are only Zariski dense. In our theorem, we need the hypothesis 
of $\Gamma$ being a lattice, because this was the case in which, so far, we have been able to prove Theorem 2.1 (cf. §1).

(3) If the $H_{i}$ 's are real algebraic, as in the case in which $H$ is real algebraic and the $\pi_{i}$ 's are rational homomorphisms, then $\operatorname{Ad}_{G}\left(H_{i}\right)$ is of finite index in a real algebraic group, so that the conclusion of the corollary is that (modulo a finite group) $\operatorname{Ad}_{G}\left(H_{1}\right)$ and $\operatorname{Ad}_{G}\left(H_{2}\right)$ are conjugate in $\operatorname{GL}(\mathfrak{g})$.

The following is just a local reformulation of the previous corollary.

Corollary 3.2'. Let $G, \Gamma, H_{i}, i=1,2$, be as above. If the $H_{1}$-action and the $H_{2}$-action on $G / \Gamma$ are smoothly isomorphic, then $\operatorname{ad}_{\mathfrak{g}}\left(\mathfrak{h}_{1}\right)$ and $\operatorname{ad}_{\mathfrak{g}}\left(\mathfrak{h}_{2}\right)$ are conjugate in $\mathfrak{g l}(\mathfrak{g})$.

Proof of Corollary 3.2. Let $\alpha_{i}: G / \Gamma \times H_{i} \rightarrow \mathrm{GL}(\mathfrak{g})$ be the derivative cocycles $\alpha_{i}\left(x, h_{i}\right)=\left(d h_{i}\right)_{x}$, for $x \in G / \Gamma$ and $h_{i} \in H_{i}$. Because of the isomorphism $\theta$ between the $H_{1}$ - and the $H_{2}$-action, we have that, for a.e. $x \in G / \Gamma$, $\theta\left(h_{1} x\right)=h_{2} \theta(x)$, where $h_{2}=\rho\left(h_{1}\right)$. Differentiation of this equality yields the relation $(d \theta)_{h_{1} x}\left(d h_{1}\right)_{x}(d \theta)_{x}^{-1}=\left(d h_{2}\right)_{\theta(x)}$ or else $\varphi\left(h_{1} x\right) \alpha_{1}\left(x, h_{1}\right) \varphi(x)^{-1}=$ $\alpha_{2}\left(\theta(x), h_{2}\right)$, where $\varphi: G / \Gamma \rightarrow \mathrm{GL}(\mathfrak{g})$ is defined as $\varphi(x)=(d \theta)_{x}$ : hence $\alpha_{2}=\Upsilon^{-1}\left(\alpha_{1}\right)$ (as defined in Proposition 2.3), so that the algebraic hulls of $\alpha_{1}$ and $\alpha_{2}$ are the same.

Proof of Theorem 3.1. As it is shown in Proposition 2.2, and with the same notation, we have the bijection

$$
H^{1}(G / \Gamma \times H ; \mathrm{GL}(n, \mathbf{R})) \leftrightarrow H^{1}(G / \Gamma \times G / H \times G ; \mathrm{GL}(n, \mathbf{R}))
$$

and

$$
H^{1}(G / H \times G / \Gamma \times G ; \mathrm{GL}(n, \mathbf{R})) \leftrightarrow H^{1}(G / H \times \Gamma ; \mathrm{GL}(n, \mathbf{R})),
$$

which, together with the obvious identification $H^{1}(G / \Gamma \times G / H \times G ; \operatorname{GL}(n, \mathbf{R}))$ $\simeq H^{1}(G / H \times G / \Gamma \times G ; \mathrm{GL}(n, \mathbf{R}))$, imply that there is a bijection in cohomology between cocycles for the $H$-action on $G / \Gamma$ and cocycles for the $\Gamma$-action on $G / H$, and this bijection preserves the algebraic hulls. However, for our purpose, we need to study the correspondence more closely; in particular, we need to see that the cocycle $\beta_{\Gamma}: G / H \times \Gamma \rightarrow \mathrm{GL}(n, \mathbf{R})$ corresponding to $\alpha: G / \Gamma \times H \rightarrow$ $\mathrm{GL}(n, \mathbf{R})$, where $\alpha(x, h)=(d h)_{x}=\operatorname{Ad}_{G}(h)$ is the adjoint cocycle, is still the restriction to $\Gamma$ of the adjoint representation of $G$. If we can prove this we are done, because then, by Theorem 2.1, the algebraic hull of $\beta_{\Gamma}: G / H \times \Gamma \rightarrow$ $\mathrm{GL}(n, \mathbf{R})$ is the same as the algebraic hull of $\beta: G / H \times G \rightarrow \mathrm{GL}(n, \mathbf{R})$ and, by transitivity of $G$ on $G / H$, this is the same as the algebraic hull of $\operatorname{Ad}_{G}(H)$ [Z5, Proposition 4.2.13]. Hence to complete the proof we need to show

Lemma 3.3. Let $H, \Gamma$ be closed subgroups of $G$, and let $\alpha: G / \Gamma \times H \rightarrow L$ be the cocycle $\alpha(x, h)=\pi(h)$ where $\pi: G \rightarrow L$ is a given homomorphism. Let $[\beta] \in H^{1}(G / H \times \Gamma ; L)$ be defined as $[\beta]=\Omega([\alpha])$, where $\Omega$ is the bijection $\Omega: H^{1}(G / \Gamma \times H ; L) \rightarrow H^{1}(G / H \times \Gamma ; L)$. Then there exists a cocycle $\beta \in[\beta]$ such that $\beta(y, \gamma)=\pi(\gamma)$. 
Proof. We shall use the same notation as in Proposition 2.2, writing, however, $H(G / \Gamma \times H)$ and $H(G / H \times \Gamma)$ for $H(G / \Gamma \times H ; L)$ and $H(G / H \times \Gamma ; L)$, respectively. Let $p: G \rightarrow G / H$ be the canonical projection, and let $\sigma: G / H \rightarrow$ $G$ be a Borel section such that $\sigma(p(g)) g \in H$ for every $g \in G$. Then we have the map

$$
\begin{array}{ccc}
\Psi: H^{1}(G / \Gamma \times H) & \rightarrow & H^{1}(G / \Gamma \times G / H \times G) \\
\alpha & \mapsto & \alpha^{0}
\end{array}
$$

defined by $\alpha^{0}(x, y, g)=\alpha\left(\sigma(y) x, \sigma(g y) g \sigma(y)^{-1}\right)=\pi\left(\sigma(g y) g \sigma(y)^{-1}\right)$, and the map

$$
\begin{array}{ccc}
\Phi: H^{1}(G / H \times G / \Gamma \times G) & \rightarrow & H^{1}(G / H \times \Gamma) \\
\beta & \mapsto & \beta_{x}
\end{array}
$$

defined by $\beta_{x}(y, \gamma)=\beta\left(g y, x, g \gamma g^{-1}\right)$, where $x=g[e]_{\Gamma} \in G / \Gamma$. Notice that $\Phi$ is independent in cohomology of the choice of $x$. Moreover, if

$$
\begin{array}{ccc}
\Sigma: H^{1}(G / \Gamma \times G / H \times G) & \rightarrow & H^{1}(G / H \times G / \Gamma \times G) \\
\alpha & \mapsto & \tilde{\alpha}
\end{array}
$$

is the identification $\Sigma(\alpha)(x, y, g)=\tilde{\alpha}(x, y, g)=\alpha(y, x, g)$, where $x \in$ $G / \Gamma, y \in G / H$, looking at the composition $\Omega=\Psi \circ \Sigma \circ \Phi$ of these maps

$$
\begin{aligned}
& H^{1}(G / \Gamma \times H) \stackrel{\Psi}{\rightarrow} H^{1}(G / \Gamma \times G / H \times G) \stackrel{\Sigma}{\rightarrow} H^{1}(G / H \times G / \Gamma \times G) \stackrel{\Phi}{\rightarrow} H^{1}(G / H \times \Gamma)
\end{aligned}
$$

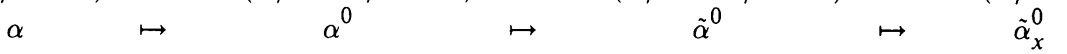

we claim that $\left[\tilde{\alpha}_{x}^{0}\right]=\left[\alpha_{\pi}\right]$. In fact if $x=g[e]_{\Gamma} \in G / \Gamma$, for a.e. $y \in G / H$ and $\gamma \in \Gamma$

$$
\begin{aligned}
\tilde{\alpha}_{x}^{0}(y, \gamma) & =\tilde{\alpha}^{0}\left(g y, x, g \gamma g^{-1}\right)=\alpha^{0}\left(x, g y, g \gamma g^{-1}\right) \\
& =\alpha\left(x \sigma(g y), \sigma(g \gamma y) g \gamma g^{-1} \sigma(g y)^{-1}\right) \\
& =\pi\left(\sigma(g \gamma y) g \gamma g^{-1} \sigma(g y)^{-1}\right) \\
& =\pi(\sigma(g \gamma y) g) \pi(\gamma) \pi\left(g^{-1} \sigma(g y)^{-1}\right) \\
& =\pi(\sigma(g \gamma y) g) \pi(\gamma) \pi(\sigma(g y) g)^{-1} \\
& =\varphi_{x}(\gamma y) \pi(\gamma) \varphi_{x}(y)^{-1},
\end{aligned}
$$

where $\varphi_{x}: G / H \rightarrow L$ defined by $\varphi_{x}(y)=\pi(\sigma(g y) g)$ is the map which implements the equivalence.

Example. We want to point out that Corollary 3.2 allows us to obtain, by completely different methods, results which were already available via other techniques. For example, if $G=\operatorname{SL}(3, \mathbf{R})$ and $\Gamma \subset G$ is any lattice, let us consider the actions on $G / \Gamma$ of the two unipotent one-parameter subgroups $H_{i}=\left\{\exp \left(t A_{i}\right): t \in \mathbf{R}\right\}$, where

$$
A_{1}=\left(\begin{array}{lll}
0 & 0 & 1 \\
0 & 0 & 0 \\
0 & 0 & 0
\end{array}\right) \text { and } A_{2}=\left(\begin{array}{ccc}
0 & 1 & 0 \\
0 & 0 & 1 \\
0 & 0 & 0
\end{array}\right) \text {. }
$$

Since $\operatorname{ad}_{\mathfrak{g}}\left(\mathfrak{h}_{1}\right)$ and $\operatorname{ad}_{\mathfrak{g}}\left(\mathfrak{h}_{2}\right)$ have different rank (as linear transformations of $\mathfrak{g})$, they cannot be conjugate in $\mathfrak{g l}(\mathfrak{g})$, thus implying that the actions of $\mathbf{R}$ by 
the identification with the $H_{i}$ 's cannot be isomorphic. The same result is a consequence of Witte's theorem [W1] since $H_{1}$ and $H_{2}$ are not conjugate in $G$ as well. (If they were, then $A_{1}$ and $A_{2}$ would be conjugate matrices in $\mathfrak{g}$, which is impossible, since they have different rank.)

Before studying actions which are just smoothly equivalent, let us examine, in contrast with what was done before, the case in which the group $G$ is compact. Since for compact groups ergodicity is equivalent to (essential) transitivity [Z5, 2.1], then the restriction of the action to a subgroup $H$ is still ergodic if and only if $H$ is dense in $G$.

Theorem 3.4. Let $H, G$ be Lie groups with $G$ compact, and let $\pi_{i}: H \rightarrow G$, $i=1,2$, be two different embeddings with dense image $\pi_{i}(H)=H_{i}$. Suppose there exists a measurable bijection $\theta: G \rightarrow G$ which conjugates the $H_{i}$-actions, $i=1,2$. Then there exists an automorphism $A$ of $G$ such that $A\left(H_{1}\right)=H_{2}$.

Remark. The same result holds also in the case in which $H_{i}$ acts on $G / G_{0}$ where $G_{0}$ is not normal in $G$.

Proof. Since the $H_{1}$-action and the $H_{2}$-action on $G$ are conjugate, we have that, for a.e. $x \in G, h_{2} \theta(x)=\theta\left(h_{1} x\right)$, where $h_{i}=\pi_{i}(h), i=1,2$. If we define a right action of $G \times G$ on $\operatorname{Map}(G, G)=\{f: G \rightarrow G \mid f$ is measurable $\}$ by $\left(f \cdot\left(g_{1}, g_{2}\right)\right)(x)=g_{2}^{-1} f\left(g_{1} x\right), \theta$ turns out to be a fixed point for the $H$ action, where $H$ acts on $\operatorname{Map}(G, G)$ via $\left(\pi_{1}, \pi_{2}\right)(H)=H_{1} \times H_{2} \subset G \times G$. By continuity of the action, $\theta$ is left fixed also by the closure $\overline{H_{1} \times H_{2}}=L$ of $H_{1} \times H_{2}$ in $G \times G$. If $p_{i}: G \times G \rightarrow G, i=1,2$, is the projection onto the $i$ th coordinate, since $H_{i} \subset p_{i}(L) \subset \bar{H}_{i}=G$, we have that $p_{i}(L)=G, i=1,2$, that is, $L$ projects onto $G$ on both coordinates. So, for $\left(g_{1}, g_{2}\right) \in L$, we have that $\left(g_{1}, g_{2}\right) \theta=\theta$, that is, $g_{2} \theta(x)=\theta\left(g_{1} x\right)$ for every $x \in G$. Notice that, since the action is free, $g_{2}$ is uniquely determined by $g_{1}$ : in other words there exists a unique $g_{2}=\lambda\left(g_{1}\right)$ such that $\left(g_{1}, \lambda\left(g_{1}\right)\right) \in L$. Moreover, since $\lambda\left(g_{1} g_{2}\right) \theta(x)=\theta\left(g_{1} g_{2} x\right)=\lambda\left(g_{1}\right) \theta\left(g_{2} x\right)=\lambda\left(g_{1}\right) \lambda\left(g_{2}\right) \theta(x)$, then $\lambda \in \operatorname{Aut}(G)$.

\section{SMOOTh ORBIT EQUiVALENCE}

If $H$ is a connected Lie group acting by diffeomorphisms on a smooth manifold $M$ with discrete stabilizers, then the $H$-orbits determine a foliation $\mathscr{F}$ on $M$ and smoothly orbit equivalent actions correspond to diffeomorphic foliations.

Theorem 4.1. Let $\mathrm{H}_{1}, \mathrm{H}_{2}$ be connected Lie groups acting by diffeomorphisms on smooth $n$-dimensional manifolds $M_{1}, M_{2}$. Suppose that the $H_{i}$-actions are essentially free, locally free and ergodic, and let $\mathscr{F}_{1}, \mathscr{F}_{2}$ be the corresponding foliations on $M_{1}, M_{2}$. If the foliations are diffeomorphic then the algebraic hulls of the derivative cocycles in the direction normal to the leaves of the foliations are conjugate. 
Proof. Since $\mathscr{F}_{1}, \mathscr{F}_{2}$ are smoothly equivalent, then there is a diffeomorphism $\theta: M_{1} \rightarrow M_{2}$ such that, for every $m \in M_{1}, h_{1} \in H_{1}, \theta\left(h_{1} m\right)=h_{2} \theta(m)$, with $h_{2}=\lambda\left(m, h_{1}\right)=\lambda_{h_{1}}(m)$, where $\lambda: M_{1} \times H_{1} \rightarrow H_{2}$ is a cocycle a.e. smooth along the orbits. If we write the previous equation as $\theta \circ L_{h_{1}}(m)=L_{\lambda\left(m, h_{1}\right)} \circ \theta(m)$, where $L_{h}(m)=h m$ denotes the action on $m$ by the diffeomorphism defined by $h$, and we let $\rho_{m_{i}}: H_{i} \rightarrow M_{i}$ denote the orbit map $\rho_{m_{i}}\left(h_{i}\right)=h_{i} m_{i}$ for $m_{i} \in M_{i}$, then differentiation of the above expression at $m$ for $h_{1} \in H_{1}$ fixed gives

$$
\begin{aligned}
(d \theta)_{h_{1} m}\left(d L_{h_{1}}\right)_{m} & =d\left(\theta \circ L_{h_{1}}\right)_{m}=d\left(L_{\lambda\left(m, h_{1}\right)} \circ \theta\right)_{m} \\
& =\left(d L_{\lambda\left(m, h_{1}\right)}\right)_{\theta(m)}(d \theta)_{m}+\left(d \rho_{\theta(m)}\right)_{\lambda\left(m, h_{1}\right)}\left(d \lambda_{h_{1}}\right)_{m},
\end{aligned}
$$

which shows that the diagram

$$
\begin{array}{cc}
T_{m} M_{1} \underset{\left(d L_{h_{1}}\right)_{m}}{\longrightarrow} T_{h_{1} m} M_{1} \\
(d \theta)_{m} \downarrow \\
T_{\theta(m)} M_{2} \underset{\left(d L_{\lambda\left(m, h_{1}\right)}\right)_{\theta(m)}}{\longrightarrow} T_{h_{2} \theta(m)} M_{2}=T_{\theta\left(h_{1} m\right)} M_{2}
\end{array}
$$

does not necessarily commute. However, the maps $L_{h_{1}}, L_{\lambda\left(m, h_{1}\right)}$ and $\theta$ preserve the foliations and their derivatives $d L_{h_{1}}, d L_{\lambda\left(m, h_{1}\right)}, d \theta$ preserve the tangent space to the foliations, that is,

$$
T_{m} \mathscr{F}_{1} \stackrel{\left(d L_{h_{1}}\right)_{m}}{\longrightarrow} T_{h_{1} m} \stackrel{\mathscr{F}_{1}}{\stackrel{(d \theta)_{h_{1} m}}{\longrightarrow}} T_{\theta\left(h_{1} m\right)} \mathscr{F}_{2}
$$

and

$$
T_{m} \mathscr{F}_{1} \stackrel{(d \theta)_{m}}{\longrightarrow} T_{\theta(m)} \mathscr{F}_{2} \stackrel{\left(d L_{\lambda\left(m . h_{1}\right.}\right)_{\theta(m)}}{\longrightarrow} T_{h_{2} \theta(m)} \mathscr{F}_{2} .
$$

Hence we have the induced diagram

$$
\begin{array}{cc}
T_{m} M_{1} / T_{m} \mathscr{F}_{1} \underset{h_{1} m}{\left(d L_{h_{1}}\right)_{m}} M_{1} / T_{h_{1} m} \mathscr{F}_{1} \\
\quad T_{\left.(d \theta)_{m}\right\rfloor} \\
T_{\theta(m)} M_{2} / T_{\theta(m)} \mathscr{F}_{2} \underset{\left(d L_{h_{1} m} m\right.}{\longrightarrow} T_{h_{2} \theta(m)} M_{2} / T_{h_{2} \theta(m)} \mathscr{F}_{2}=T_{\theta\left(h_{1} m\right)} M_{2} / T_{\theta\left(h_{1} m\right)} \mathscr{F}_{2}
\end{array}
$$

which, if we denote, by $N_{m} \mathscr{F}_{i}=T_{m} M_{i} / T_{m} \mathscr{F}_{i}$, the normal space to the foliation $\mathscr{F}_{i}$ at the point $m$, becomes

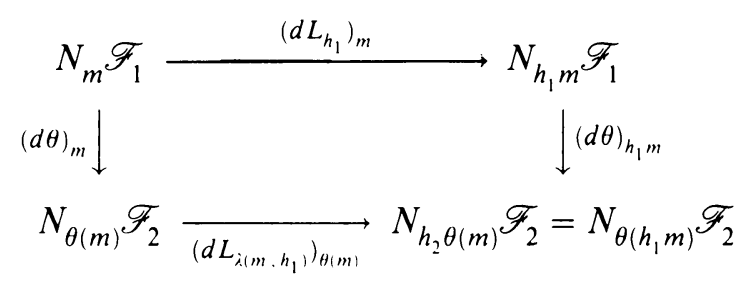


Now we need the following

Lemma 4.2. $\left.\left(d \lambda_{h_{1}}\right)\right|_{N_{m} \mathscr{F}_{1}}=0$ and the diagram (*) commutes.

Assuming this lemma, whose proof we postpone for a while, we have that $\left.\left.(d \theta)\right|_{N_{h_{1} m} \mathscr{F}_{1}}\left(d L_{h_{1}}\right)\right|_{N_{m} \mathscr{F}_{1}}=\left.\left.\left(d L_{\lambda\left(m, h_{1}\right)}\right)\right|_{N_{\theta(m)} \mathscr{F}}(d \theta)\right|_{N_{m} \mathscr{F}_{1}}=\left.\left.\left(d L_{h_{2}}\right)\right|_{N_{\theta(m)} \mathscr{F}_{2}}(d \theta)\right|_{N_{m} \mathscr{F}_{1}}$, that is,

$$
\left.\left.\left.(d \theta)\right|_{N_{h_{1} m} \mathscr{F}_{1}}\left(d L_{h_{1}}\right)\right|_{N_{m} \mathscr{F}_{1}}(d \theta)^{-1}\right|_{N_{m} \mathscr{F}_{1}}=\left.\left(d L_{h_{2}}\right)\right|_{N_{\theta(m)} \mathscr{F}_{2}} \cdot
$$

Using again Proposition 2.3 we can easily deduce that the algebraic hulls of $\left.\left(d L_{h_{1}}\right)\right|_{N_{m} \mathscr{F}_{1}}$ and $\left.\left(d L_{h_{2}}\right)\right|_{N_{\theta(m)} \mathscr{F}_{2}}$ are the same. In fact, if $k$ and $n(k<n)$ are the dimensions of the foliations and the manifolds respectively, let $\alpha_{i} \in$ $Z\left(M_{i} \times H_{i} ; \mathrm{GL}(n-k, \mathbf{R})\right)$ be defined as $\alpha_{i}\left(m_{i}, h_{i}\right)=\left.\left(d L_{h_{i}}\right)\right|_{N_{m_{i}} \mathscr{F}_{i}}, i=1,2$. Since, by Proposition 2.3 (and using the same notation), we have that the algebraic hulls of $\Upsilon\left(\alpha_{2}\right)$ and $\alpha_{2}$ are the same, it will be enough to show that $\Upsilon\left(\alpha_{2}\right)=\alpha_{1}$ in cohomology. In fact

$$
\begin{aligned}
\Upsilon\left(\alpha_{2}\right)\left(m, h_{1}\right) & =\alpha_{2}\left(\theta(m), \lambda\left(m, h_{1}\right)\right)=\left.\left(d L_{h_{2}}\right)\right|_{N_{\theta(m)} \mathscr{F}_{2}} \\
& =\left.\left.\left.(d \theta)\right|_{N_{h_{1} m} \mathscr{F}_{1}}\left(d L_{h_{1}}\right)\right|_{N_{m} \mathscr{F}_{1}}(d \theta)^{-1}\right|_{N_{m} \mathscr{F}_{1}} \\
& =\left.\left.(d \theta)\right|_{N_{h_{1} m} \mathscr{F}_{1}} \alpha_{1}\left(m, h_{1}\right)(d \theta)^{-1}\right|_{N_{m} \mathscr{F}_{1}}=\alpha_{1}^{\prime}\left(m, h_{1}\right),
\end{aligned}
$$

where $\alpha_{1} \sim \alpha_{1}^{\prime}$ via the map $\phi: M_{1} \rightarrow \mathrm{GL}(n-k, \mathbf{R})$ defined as $\phi(m)=$ $\left.(d \theta)\right|_{N_{m} \mathscr{F}_{1}}$. Hence $\left[\Upsilon\left(\alpha_{2}\right)\right]=\left[\alpha_{1}\right]$ and this completes the proof.

Proof of Lemma 4.2. We want to differentiate the equation $\theta\left(h_{1} m\right)=h_{2} \theta(m)$ in local coordinates. Observe first of all that we can choose coordinate charts $\left\{\left(U_{m}, \varphi_{m}\right)\right\}_{m \in M_{1}},\left\{\left(V_{m^{\prime}}, \psi_{m^{\prime}}\right)\right\}_{m^{\prime} \in M_{2}}$ on $M_{1}$ and $M_{2}$ such that the map $\theta: M_{1}$ $\rightarrow M_{2}$ is locally just the identity map. In fact let $\left\{\left(U_{m}, \varphi_{m}\right)\right\}_{m \in M_{1}}$ be charts which realize the foliation $\mathscr{F}_{1}$, namely, $\varphi_{m}\left(\left(H_{1}\right.\right.$-orbit $\left.) \cap U_{m}\right)=\left\{\mathbf{R}^{k} \times \varphi_{m}\left(U_{m}\right)\right\} \times$ $\{p t\}$, and assume for simplicity that $\varphi_{m}(m)=(0,0)$. We can choose charts $\left\{\left(V_{m^{\prime}}, \psi_{m^{\prime}}\right)\right\}$ on $M_{2}$ defined by $\left(V_{m^{\prime}}, \psi_{m^{\prime}}\right)=\left(V_{\theta(m)}, \psi_{\theta(m)}\right)=\left(\theta\left(U_{m}\right), \varphi_{m} \circ\right.$ $\left.\theta^{-1}\right)$, and it is straightforward to check that these charts realize the foliation $\mathscr{F}_{2}, \psi_{m^{\prime}}\left(m^{\prime}\right)=\left(\varphi_{m} \circ \theta^{-1}\right)(\theta(m))=(0,0)$ and that, locally, the map $\theta_{m}: \varphi_{m}\left(U_{m}\right) \rightarrow \psi_{\theta(m)}\left(V_{\theta(m)}\right)=\varphi_{m}\left(U_{m}\right)$ defined by $\theta_{m}=\psi_{\theta(m)} \circ \theta \circ \varphi_{m}^{-1}$ is just the identity. Fix $m_{0} \in M_{1}$; if $m \in U_{m_{0}}, h_{1} \in H_{1}$, we have $h_{1} m \in$ $U_{h_{1} m_{0}}$ so that $\varphi_{m_{0}}(m)=(x, y)$ and $\varphi_{h_{1} m_{0}}\left(h_{1} m\right)=\left(\tau_{h_{1}}(x, y), \boldsymbol{\xi}(y)\right)$, where $\tau_{h_{1}}: \mathbf{R}^{k} \times \mathbf{R}^{n-k} \rightarrow \mathbf{R}^{k}$ is just the map which describes in local coordinates the translation of $m$ along the fiber by $h_{1} \in H_{1}$ (i.e., $\tau_{h_{1}}=L_{h_{1}}$ in local coordinates) and $\xi: \mathbf{R}^{n-k} \rightarrow \mathbf{R}^{n-k}$ indicates in local coordinates the fiber of the point $h_{1} m$ (notice that if $h_{1} \in H_{1}$ is close enough to the identity so that $h_{1} m \in U_{m_{0}}$, then $\xi(y)=y$ ). Moreover, because of our choice of charts on $M_{2}$, since 
$\theta(m) \in \theta\left(U_{m_{0}}\right)=V_{\theta\left(m_{0}\right)}$ and $h_{2} \theta(m)=\theta\left(h_{1} m\right) \in \theta\left(U_{h_{1} m_{0}}\right)=V_{\theta\left(h_{1} m_{0}\right)}$, we can write $\psi_{\theta\left(m_{0}\right)}(\theta(m))=(x, y)$ and $\psi_{\theta\left(h_{1} m_{0}\right)}\left(h_{2} \theta(m)\right)=\left(\tau_{h_{2}}(x, y), \eta(y)\right)$, where $\tau_{h_{2}}, \eta$ describe in local coordinates (as observed above for $\tau_{h_{1}}$ and $\xi$ ) the action of $h_{2}$ on $\theta(m)$ and, here, $h_{2}=\lambda\left(m, h_{1}\right)$ is a function of $m$. Then these equations together give us that

$$
\begin{aligned}
\left(\tau_{h_{2}}(x, y), \eta(y)\right) & =\psi_{\theta\left(h_{1} m_{0}\right)}\left(h_{2} \theta(m)\right)=\psi_{\theta\left(h_{1} m_{0}\right)}\left(\theta\left(h_{1} m\right)\right) \\
& =\psi_{\theta\left(h_{1} m_{0}\right)}\left(\theta\left(\varphi_{h_{1} m_{0}}^{-1}\left(\tau_{h_{1}}(x, y), \xi(y)\right)\right)\right) \\
& =\theta_{h_{1} m_{0}}\left(\tau_{h_{1}}(x, y), \xi(y)\right)=\left(\tau_{h_{1}}(x, y), \xi(y)\right) .
\end{aligned}
$$

From these equalities it follows that $\xi=\eta$ and

$$
\left(\tau_{h_{2}}(x, y), \xi(y)\right)=\left(\tau_{h_{1}}(x, y), \xi(y)\right) .
$$

Differentiation of this equality, using the notation

$$
\tau_{h_{j}}^{i}(x, y)=\left.\frac{\partial \tau_{h_{j}}\left(u_{1}, u_{2}\right)}{\partial u_{i}}\right|_{(x, y)} \text { and } \tau_{h_{2}}(x, y)=\zeta_{(x, y)}\left(h_{2}\right)
$$

(i.e., $\zeta_{(x, y)}=\rho_{m}$ in local coordinates), gives

$$
\begin{aligned}
\left(\begin{array}{cc}
\tau_{h_{1}}^{1}(x, y) & \tau_{h_{1}}^{2}(x, y) \\
0 & \frac{\partial \xi}{\partial y}
\end{array}\right) & =\left(\begin{array}{cc}
\tau_{h_{2}}^{1}(x, y)+\frac{d \zeta_{(x, y)} \frac{\partial \lambda}{\partial h_{2}}}{\partial x} & \tau_{h_{2}}^{2}(x, y)+\frac{d \zeta_{(x, y)} \frac{\partial \lambda}{\partial h_{2}}}{\partial y} \\
0 & \frac{\partial \xi}{\partial y}
\end{array}\right) \\
& =\left(\begin{array}{cc}
\tau_{h_{2}}^{1}(x, y) & \tau_{h_{2}}^{2}(x, y) \\
0 & \frac{\partial \xi}{\partial y}
\end{array}\right)+\frac{d \zeta_{(x, y)}}{d h_{2}}\left(\begin{array}{cc}
\frac{\partial \lambda}{\partial x} & \frac{\partial \lambda}{\partial y} \\
0 & 0
\end{array}\right),
\end{aligned}
$$

which is nothing but the expression in local coordinates of the equation

$$
(d \theta)_{h_{1} m}\left(d L_{h_{1}}\right)_{m}=\left(d L_{\lambda\left(m, h_{1}\right)}\right)_{\theta(m)}(d \theta)_{m}+\left(d \rho_{\theta(m)}\right)_{\lambda\left(m, h_{1}\right)}\left(d \lambda_{h_{1}}\right)_{m} .
$$

But this shows that $\left.(d \lambda)\right|_{N_{m} F_{1}}=0$ which implies the commutativity of the diagram $(*)$.

In order to apply Theorem 4.1 we need the following

Proposition 4.3. Let

$$
G(k, n-k)=\left(\begin{array}{cc}
\mathrm{GL}(k, \mathbf{R}) & * \\
0 & \mathrm{GL}(n-k, \mathbf{R})
\end{array}\right) \subset \mathrm{GL}(n, \mathbf{R}),
$$

and consider the surjective homomorphism $p: G(k, n-k) \rightarrow \mathrm{GL}(n-k, \mathbf{R})$. Suppose $\alpha: G \times S \rightarrow G(k, n-k)$ is a cocycle with algebraic hull $H \subseteq$ $G(k, n-k)$. Then the algebraic hull of $p \circ \alpha$ is the Zariski closure of $p(H)$ in which $p(H)$ is of finite index.

Proof. Since $p$ is surjective, the algebraic hull of $p \circ \alpha$ cannot be strictly contained in $p(H)$. Let $H_{\mathbf{C}}$ be a complex algebraic group defined over $\mathbf{R}$ such that $H=\left(H_{\mathbf{C}}\right)_{\mathbf{R}}$ consists of its real points. Since $p$ is $\mathbf{R}$-regular, we have that $p\left(H_{\mathbf{C}}\right)$ is an $\mathbf{R}$-algebraic group, but $p(H)$ is not necessarily Zariski closed. However, 
if we denote by $\overline{p(H)}^{Z}$ the Zariski closure of $p(H)$ in $\operatorname{GL}(n-k, \mathbf{R})$, we have the inclusions $p(H) \subset \overline{p(H)}^{Z} \subset\left(p\left(H_{\mathbf{C}}\right)\right)_{\mathbf{R}}$ : since $p(H)=p\left(\left(H_{\mathbf{C}}\right)_{\mathbf{R}}\right)$ is of finite index in $\left(p\left(H_{\mathbf{C}}\right)\right)_{\mathbf{R}}$, it follows that $p(H)$ is of finite index in $\overline{p(H)}^{Z}$ as well.

The main application for the theorem about smooth orbit equivalence that we present here is to the $H_{i}$-action on $G / \Gamma$, and we shall illustrate with an example at the end of this section how to obtain results which did not seem to be accessible by other methods. However, before doing this we want to show how, in this case, essential freeness follows almost automatically.

Proposition 4.4. Let $G$ be a noncompact connected simple Lie group, $\Gamma \subset G$ a lattice and $H \subset G$ an almost connected real algebraic group. Then $H$ acts essentially freely on $G / \Gamma$.

Remark. For simplicity we stated the result only for simple groups; however, exactly the same proof works in the case in which $G$ is semisimple and $\Gamma \subset G$ is irreducible, as long as we assume that $\Gamma$ does not intersect a subgroup $N \subseteq H$ normal in $G$. Moreover, small modifications of the proof show that the result holds also in the case in which $H$ is not algebraic.

We start the proof with the following lemma.

Lemma 4.5. Essential freeness of $H$ acting on $G / \Gamma$ is equivalent to essential freeness of $\Gamma$ acting on $G / H$.

Proof. This is just a straightforward exercise on double cosets. If $\mu$ is the Haar measure on $G$, let $\mu_{\Gamma}$ and $\mu_{H}$ be the measures respectively on $G / \Gamma$ and $G / H$. Notice that, since $G$ is connected and $H$ is a proper subgroup of smaller dimension, if $X \subset G$ is a measurable subset then $\mu_{\Gamma}\left(p_{\Gamma}(X)\right)=0$ if and only if $\mu(X)=0$ if and only if $\mu_{H}\left(p_{H}(X)\right)=0$, where we denote with $p_{\Gamma}: G \rightarrow G / \Gamma$ and $p_{H}: G \rightarrow G / H$ the canonical projections. Let $W=$ $\left\{x \Gamma: \operatorname{Stab}_{H}(x \Gamma) \neq e\right\} \subseteq G / \Gamma$ and $V=\left\{x H: \operatorname{Stab}_{\Gamma}(x H) \neq e\right\} \subseteq G / H$. We want to show that $\mu_{H}(V)=0$ if and only if $\mu_{\Gamma}(W)=0$. An easy computation shows that, for $x \in G, x \Gamma \in W$ if and only if $x^{-1} H \in V$, so that $p_{H}\left(\left(\sigma_{\Gamma}(W)\right)^{-1}\right) \subseteq V$, where $\sigma_{\Gamma}: G / \Gamma \rightarrow G$ is a section of the $p_{\Gamma}$. Hence $\mu_{H}\left(p_{H}\left(\left(\sigma_{\Gamma}(W)\right)^{-1}\right)\right) \leq \mu_{H}(V)$. If $\mu_{H}(V)=0$ then $\mu_{H}\left(p_{H}\left(\left(\sigma_{\Gamma}(W)\right)^{-1}\right)\right)=0$ so that $0=\mu\left(\sigma_{\Gamma}(W)\right)^{-1}=\mu\left(\sigma_{\Gamma}(W)\right)=\mu_{\Gamma}(W)$ which is what we wanted: the other direction (which actually we shall not use), follows by symmetry.

Proof of 4.4. Because of the lemma, it is enough to show that $\Gamma$ is essentially free on $G / H$. With the notation used in 4.5 we need to show that $\mu_{H}(V)=0$. For each $\gamma \in \Gamma$, let $V_{\gamma}=\{x H \in G / H: \gamma x H=x H\}$; it is clear that $V_{\gamma}$ is a subvariety and that $V=\bigcup_{\gamma \in \Gamma} V_{\gamma}$, where the union is countable. Then it will be enough to show that, for every $\gamma \in \Gamma, V_{\gamma}$ is a subvariety of smaller dimension, so that $\mu_{H}\left(V_{\gamma}\right)=0$. Since $G$ is connected, $G / H$ is an irreducible variety, so that if $V_{\gamma}$ does not have smaller dimension it must be $V_{\gamma}=G / H$. But if, for 
some $\gamma_{0} \in \Gamma, \gamma_{0} \neq e$, we have $V_{\gamma_{0}}=G / H$, then, since $G / H=V_{\gamma_{0}}=g V_{\gamma_{0}}=$ $V_{g \gamma_{0} g^{-1}}$ for any $g \in G$, this would imply the existence of a connected normal subgroup $N=G \gamma_{0} G^{-1}$ which is proper (since it fixes the whole space, hence $N \subseteq H$ ) and this is impossible since $G$ is simple.

Example. Let $H=\mathbf{R}^{\times} \times \mathbf{R}$ be the group of the affine motions of the line. If $\Gamma \subset \operatorname{SL}(3, \mathbf{R})$ is any lattice let us consider the $H$-action on $\operatorname{SL}(3, \mathbf{R}) / \Gamma$ by $\pi_{1}, \pi_{2}: H \hookrightarrow \operatorname{SL}(3, \mathbf{R})=G$, defined by

$$
\pi_{1}(\lambda, t)=\left(\begin{array}{ccc}
\lambda & 0 & t \\
0 & \lambda^{-1} & 0 \\
0 & 0 & 1
\end{array}\right) \quad \text { and } \pi_{2}(\lambda, t)=\left(\begin{array}{ccc}
\lambda & 0 & t \\
0 & \lambda & 0 \\
0 & 0 & \lambda^{-2}
\end{array}\right) .
$$

We claim that these two actions cannot be smoothly orbit equivalent, and we shall prove it by showing that $\left.\operatorname{Ad}_{G}\left(H_{1}\right)\right|_{\mathfrak{g} / \mathfrak{h}_{1}}$ and $\left.\operatorname{Ad}_{G}\left(H_{2}\right)\right|_{\mathfrak{g} / \mathfrak{h}_{2}}$ are not conjugate in $\mathrm{GL}(\mathfrak{g})$. In fact, because of Theorem 3.1, we have that the algebraic hull of the $H_{i}$-action on $G / \Gamma$ is the Zariski closure of $\operatorname{Ad}_{G}\left(H_{i}\right)$, and Proposition 4.3 implies that the algebraic hull of the $H_{i}$-action in the direction normal to the orbits is the Zariski closure of $\left.\operatorname{Ad}_{G}\left(H_{i}\right)\right|_{\mathfrak{g} / \mathfrak{h}_{i}}$. Let $C_{\mathfrak{g} / \mathfrak{h}_{i}}\left(h_{i}\right)$ be the centralizer of $h_{i} \in H_{i}$ in $\mathfrak{g} / \mathfrak{h}_{i}$ and $V_{h_{i}}=\left\{X \in \mathfrak{g} / \mathfrak{h}_{i}:\left.\operatorname{Ad}_{G}\left(h_{i}\right)\right|_{\mathfrak{g} / \mathfrak{h}_{i}} X \stackrel{=}{=}\right\}$. Then we have that $C_{\mathfrak{g} / \mathfrak{h}_{i}}\left(h_{i}\right)=V_{h_{i}}$, for $h_{i} \in H_{i}$, so that

$$
C_{\mathfrak{g} / \mathfrak{h}_{i}}\left(H_{i}\right)=\bigcap_{h_{i} \in H_{i}} C_{\mathfrak{g} / \mathfrak{h}_{i}}\left(h_{i}\right)=\bigcap_{h_{i} \in H_{i}} V_{h_{i}}=V_{H_{i}} .
$$

An easy computation shows that $C_{\mathfrak{g} / \mathfrak{h}_{i}}\left(H_{1}\right)$ (and hence $V_{H_{1}}$ ) is zero-dimensional and $C_{\mathfrak{g} / \mathfrak{h}_{2}}\left(H_{2}\right)$ (and hence $V_{H_{2}}$ ) consists of matrices of the form

$$
\left\{\left(\begin{array}{lll}
0 & a & 0 \\
0 & 0 & 0 \\
0 & 0 & 0
\end{array}\right): a \in \mathbf{R}\right\} .
$$

Since $\left.\operatorname{Ad}_{G}(H)\right|_{\mathfrak{g} / \mathfrak{h}_{i}}$ is of finite index in its Zariski closure and since the dimension of the subspace of fixed vectors would be an invariant of conjugacy, it follows that the algebraic hulls of $\left.\operatorname{Ad}_{G}\left(H_{1}\right)\right|_{\mathfrak{g} / \mathfrak{h}_{1}}$ and $\left.\operatorname{Ad}_{G}\left(H_{2}\right)\right|_{\mathfrak{g} / \mathfrak{h}_{2}}$ cannot be conjugate in $\mathrm{GL}(\mathfrak{g})$.

\section{REFERENCES}

[Be] D. Benardete, Topological equivalence of flows on homogeneous spaces and divergence of one-parameter subgroups of Lie groups, Trans. Amer. Math. Soc. 306 (1988), 499-528.

[CFW] A. Connes, J. Feldman, and B. Weiss, An amenable equivalence relation is generated by a single transformation, Ergodic Theory Dynamical Systems 1 (1981), 431-450.

[D1] H. A. Dye, On groups of measure preserving transformations. I, Amer. J. Math. 81 (1959), 119-159.

[D2] - - On groups of measure preserving transformations. II, Amer. J. Math. 85 (1963), 551-

[I] A. Iozzi, Invariant geometric structures: a non-linear extension of the Borel density theorem, Amer. J. Math. (to appear). 
[K] W. Krieger, On ergodic flows and the isomorphism of factors, Math. Ann. 223 (1976), 19-70.

[M] B. Marcus, Topological conjugacy of horocycle flows, Amer. J. Math. 88 (1966), 154-178.

[PnZ] P. Pansu and R. J. Zimmer, Rigidity of locally homogeneous metrics of negative curvature on the leaves of a foliation, Israel J. Math. 68 (1989), 56-62.

[Pr] W. Parry, Metric classification of ergodic nilflows, Amer. J. Math. 88 (1966), 819-829.

[Rm] A. Ramsay, Virtual groups and group actions, Adv. in Math. 6 (1971), 253-322.

[Rt] M. Ratner, Ergodic theory in hyperbolic space, Conference on Modern Analysis and Probability (New Haven, Conn., 1982), R. Beals, A. Beck, A. Bellow and A. Hajian, eds., Contemp. Math., vol. 26, Amer. Math. Soc., Providence, R.I., 1984.

[W1] D. Witte, Zero-entropy affine maps on homogeneous spaces, Amer. J. Math. 109 (1987), 927-961.

[W2] _ Topological equivalence of foliations of homogeneous spaces, Trans. Amer. Math. Soc. 317 (1990), 143-166.

[Z1] R. J. Zimmer, Orbit spaces of unitary representations, ergodic theory and simple Lie groups, Ann. of Math. 106 (1977), 573-588.

[Z2] _ Strong rigidity for ergodic actions of semisimple Lie groups, Ann. of Math. 112 (1980), 511-529.

[Z3] _- On the cohomology of ergodic group actions, Israel J. Math. 35 (1980), 289-300.

[Z4] _ Orbit equivalence and rigidity of ergodic actions of Lie groups, Ergodic Theory Dynamical Systems 1 (1981), 237-253.

[Z5] _ Ergodic theory and semisimple groups, Birkhäuser, Boston, Mass., 1984.

[Z6] __ Ergodic theory and the automorphism group of a G-structure, Group Representations, Ergodic Theory, Operator Algebras, and Mathematical Physics, Ed., C. C. Moore, SpringerVerlag, New York, 1987.

Department of Mathematics, University of Chicago, Chicago, Illinois 60637

Current address: Department of Mathematics, University of Pennsylvania, David Rittenhouse Laboratory, 209 South 33rd Street, Philadelphia, Pennsylvania 19104-6395

E-mail address: IOZZI@NEXT.SAS.UPENN.EDU 